[7] - Propriétés des points extrémaux des ensembles plans et leur application à la représentation conforme, ibidem 3 (1957), p. $319-342$.

[8] - Sur une classe de fonctions homogènes et les séries de Taylor des fonctions de deux variables, Annales de la Société Polonaise de Mathématiques 23 (1956), p. $245-268$.

[9] J. Siciak, On some applications of the method of extremal points, Colloquium Mathematicum 11 (1964), p. $209-250$.

\section{EXTREMAL POINTS IN THE SPACE $C^{n}$}

$\mathrm{BY}$

J. SICIAK (CRACOW)

This is a report* on an extension of Leja's method to problems in several complex variables.

1. Interpolation formulas. The Lagrange interpolation formulas for ordinary polynomials of $n$ variables and for homogeneous polynomials of $n$ variables are basic tools in the method of extremal points in the space $C^{n}$ of $n$ complex variables.

Any polynomial $P_{v}(z)$ of degree $v$ may be written in the form

$$
P_{v}(z)=\sum_{l=1}^{v_{*}^{*}} a_{k_{1 l} k_{2 l} \ldots k_{n l}} z_{1}^{k_{1} l l} z_{2}^{k_{2 l}} \ldots z_{n}^{k_{n l}}
$$

where $\left(k_{1 l}, k_{2 l}, \ldots, k_{n l}\right), \quad l=1,2, \ldots, v_{*}, v_{*}=\left(\begin{array}{c}\nu+n \\ n\end{array}\right)$, is the sequence of all solutions in non-negative integers of the inequality $k_{1}+k_{2}+\ldots+k_{n} \leqslant v$. Analogously, any homogeneous polynomial $Q_{v}(z)$ of degree $v$ may be written in the form

$$
Q_{\nu}(z)=\sum_{l=1}^{v_{0}} a_{h_{1 l} h_{2 l} \ldots h_{n l}} z_{1}^{h_{1 l}} z_{2}^{h_{2 l}} \ldots z_{n}^{h_{n l}}
$$

where $\left(h_{1 l}, h_{2 l}, \ldots, h_{n l}\right), l=1,2, \ldots, v_{0}, v_{0}=\left(\begin{array}{c}v+n-1 \\ n-1\end{array}\right)$, is a complete sequence of the solutions in non-negative integers of the equation $h_{1}+$ $+h_{2}+\ldots+h_{n}=\nu$.

Suppose $p^{(v)}=\left(p_{1}, p_{2}, \ldots, p_{v_{*}}\right)$ is a system of $\nu_{*}$ points $p_{i}=\left(z_{1 i}, \ldots\right.$ $\left.\ldots, z_{n i}\right), i=1,2, \ldots, v_{*}$, of $C^{n}$ such that the determinant $V\left(p^{(v)}\right)=$ $=V\left(p_{1}, \ldots, p_{v_{*}}\right)$ defined by

$$
\text { (1.3) } \quad V\left(p^{(v)}\right)=\operatorname{det}\left[z_{1 i}^{k_{11} l} z_{2 i}^{k_{2 l} l} \ldots z_{n_{i}}^{k_{n l}}\right], \quad i, l=1,2, \ldots, v_{*} \text {, }
$$

is different from zero. Then the following interpolation formula holds:

$$
P_{\nu}(z)=\sum_{i=1}^{\nu_{*}} P_{\nu}\left(p_{i}\right) L^{(i)}\left(z, p^{(\nu)}\right), \quad z \epsilon C^{n},
$$

* Presented to the Third Conference on Analytic Functions, held in Cracow, 30. VIII - 4. IX. 1962 
where

$$
L^{(i)}\left(z, p^{(v)}\right)=V\left(p_{1}, \ldots, p_{i-1}, z, p_{i+1}, \ldots, p_{\nu_{*}}\right) / V\left(p^{(v)}\right) .
$$

Analogously, if $p^{(v)}=\left(p_{1}, \ldots, p_{v_{0}}\right)$ is a system of $v_{0}$ points of $C^{n}$ such that the determinant

(1.6) $W\left(p^{(v)}\right)=W\left(p_{1}, \ldots, p_{v_{0}}\right)=\operatorname{det}\left[z_{1 i}^{h_{1} l} z_{2 i}^{h_{2 l}} \ldots z_{n_{i}}^{h_{n l}}\right] \quad\left(i, l=1,2, \ldots, v_{0}\right)$ does not vanish, then

$$
Q_{\nu}(z)=\sum_{i=1}^{\nu_{0}} Q_{\nu}\left(p_{i}\right) T^{(i)}\left(z, p^{(\nu)}\right), \quad z \epsilon C^{n},
$$

where

$$
T^{(i)}\left(z, p^{(\nu)}\right)=W\left(p_{1}, \ldots, p_{i-1}, z, p_{i+1}, \ldots, p_{v_{0}}\right) / W\left(p^{(\nu)}\right) .
$$

2. Extremal points. Let $E$ be a bounded closed set in $C^{n}$ and let $b(z)$ be a real function bounded and lowersemicontinuous in $E$. Given an arbitrary system $p^{(v)}=\left(p_{1}, p_{2}, \ldots, p_{\nu_{*}}\right) \in E$, we define $\nabla\left(p^{(\nu)}, b\right)$ by

$$
\nabla\left(p^{(\nu)}, b\right)=V\left(p^{(\nu)}\right) \exp \left(-\nu \sum_{i=1}^{\nu_{*}} b\left(p_{i}\right)\right), \quad \nu=1,2, \ldots
$$

A system

$$
q^{(\nu)}=\left(q_{1}^{(\nu)}, \ldots, q_{\nu_{*}}^{(\nu)}\right)
$$

such that

$$
\nabla\left(q^{(v)}, b\right)=\max _{v^{(v)} \in E}\left|\nabla\left(p^{(v)}, b\right)\right|
$$

is called the $\nu$-th extremal system of $E$ with respect to $b(z)$ and the determinant $V\left(p^{(\nu)}\right)$.

Given an arbitrary system $p^{(\nu)}=\left(p_{1}, \ldots, p_{v_{0}}\right) \in E$, we define $W\left(p^{(\nu)}, b\right)$ by

\section{A system}

$$
W\left(p^{(v)}, b\right)=W\left(p^{(v)}\right) \exp \left(-v \sum_{i=1}^{v_{0}} b\left(p_{i}\right)\right)
$$

such that

$$
h^{(v)}=\left(h_{1}^{(v)}, \ldots, h_{\nu_{0}}^{(\nu)}\right)
$$

$$
W\left(h^{(v)}, b\right)=\max _{p^{(v)} \in \mathbb{E}}\left|W\left(p^{(v)}, b\right)\right|
$$

is called the $\nu$-th extremal system of $E$ with respect to $b(z)$ and the determinant $W\left(p^{(v)}\right)$.
Let us define $v_{v}(E, b)$ and $w_{v}(E, b)$ by

$$
\begin{aligned}
& v_{\nu}(E, b)=\nabla\left(q^{(v)}, b\right)^{1 / n\left(\begin{array}{c}
\nu+n \\
n+1
\end{array}\right),} \\
& w_{\nu}(E, b)=W\left(h^{(\nu)}, b\right)^{1 / v\left(\begin{array}{c}
\nu+n-1 \\
n-1
\end{array}\right)}
\end{aligned}
$$

The extremal points (2.2) have been introduced by Fekete [2] in the case of $E \subset C^{1}$ and $b(z) \equiv 0$. In the case of $E \subset C^{1}$, they have been introduced by Leja [8] and investigated latter by him and his students in connection with the Dirichlet problem and conformal mapping (see report [5] by Górski).

In the case of $n=2$ the extremal points (2.5) have been introduced by Leja [6] (for $b(z) \equiv 0$ ) and applied by him to the investigation of domains of uniform convergence of a series of homogeneous polynomials of two complex variables ([6], [9]). Points $(2.5)(b(z) \equiv 0)$ heve been exploited to the same purpose in the case of $C^{n}, n \geqslant 3$, in [15].

It is known ([6], [7], [8], [10]) that the sequence $\left\{v_{p}(E, b)\right\}, E \subset C^{1}$, and the sequence $\left\{w_{\nu}(E, b)\right\}, E \subset C^{2}$, are both convergent. The limit $v(E, 0)=\lim v_{v}(E, 0)$ is called the transfinite diameter of $E$. The limit $w(E, 0)=\lim _{\nu \rightarrow \infty} w_{\nu}(E, 0), E \subset C^{2}$, is called the triangular ecart of $E([6],[9])$.

The question (formulated by Leja [11] in a slightly different form) as to whether the sequences (2.7) or (2.8) for $E \subset C^{n}, n \geqslant 2, b(z) \equiv 0$, are convergent or not remains still unsolved (except for $E=E_{1} \times E_{2} \times$ $\times \ldots \times E_{n} ;$ see $\left.[14]\right)$.

3. Extremal functions $\Phi(z, E, b)$ and $\psi(z, E, b)$. Define

and

$$
\Phi_{v}(z, E, b)=\max _{(i)}\left(\left|L^{(i)}\left(z, q^{(\nu)}\right)\right| \exp \left[v b\left(q_{i}^{(v)}\right)\right]\right)
$$

(3.2) $\quad \psi_{v}(z, E, b)=\max _{(i)}\left(\left|T^{(i)}\left(z, h^{(\nu)}\right)\right| \exp \left[\nu b\left(h_{i}^{(\nu)}\right)\right]\right), \quad v=1,2, \ldots$

THEOREM. The sequences $\left\{\sqrt[y]{\Phi_{v}(z, E, b)}\right\}$ and $\left\{\sqrt[y]{\psi_{\nu}(z, E, b)}\right\}$ are convergent at any finite point $z \in C^{n}$ to the limits $\Phi(z, E, b)$ and $\psi(z, E, b)$, respectively (the limits being finite or not),

$$
\begin{gathered}
\Phi(z, E, b)=\lim _{\nu \rightarrow \infty} \sqrt[v]{\Phi_{\nu}(z, E, b)}, \\
\psi(z, E, b)=\lim _{\nu \rightarrow \infty} \sqrt[v]{\psi_{\nu}(z, E, b)} .
\end{gathered}
$$

If $E$ contains a subset $F=F_{1} \times \ldots \times F_{n}$ such that the transfinite diameter $d\left(F_{i}\right)$ of $F_{i}(i=1,2, \ldots, n)$ is positive, then the functions $\Phi$ and $\psi$ are bounded in every compact subset of $C^{n}$. In the case of $C^{1}$ the function $\Phi$ has been introduced by Leja [8] (for more details see report [5] by Górski) and in the general case by Siciak [16]. The function $\psi$ 
has been introduced by Leja [6] in the case of $C^{2}$ and $b(z) \equiv 0$, and by Siciak [15], [16] in the general case. Leja defines $\Phi$ and $\psi$ as limits of some sequences different from (3.1) and (3.2) and his method of proof of the existence of the limits is based on the fact that every ordinary polynomial in the space $C^{1}$ or every homogeneous polynomial in $C^{2}$ is a product of linear factors. This fact no longer holds in higher dimensional spaces. The method used by Siciak is based on the interpolation formulas (1.4) or (1.7) and does not depend on the dimension.

4. An application of $\psi(z, E, b)$. The usefulness of the function $\psi(z, E, b)$ is conspicuously due to the following theorems (see [6], [9], and $[15])$.

(i) If

$$
\sum_{\nu=0}^{\infty} Q_{\nu}(z)
$$

is a series of homogeneous polynomials $Q_{\nu}$ of respective degrees $\nu$ uniformly bounded on $E$, then the series (4.1) converges absolutely at least in the set

$$
G(E)=(z: \psi(z, E, 0)<1) .
$$

(ii) If $\psi(z, E, 0)$ is bounded in every compact subset of $C^{n}$, then $G(E)$ contains interior points. The interior $\stackrel{\circ}{G}(E)$ of $G(E)$ is given by

$$
\stackrel{\circ}{G}(E)=\left(z: \psi^{*}(z, E, 0)<1\right),
$$

where $\psi^{*}(z, E, 0)=\limsup _{z^{\prime} \rightarrow z} \psi\left(z^{\prime}, \pm, 0\right)$. Moreover, if $n=2$, the necessary and sufficient condition set of $C^{n}$, is that $E$ have a positive triangular ecart.

(iii) There exists a series of homogeneous polynomials with terms uniformly bounded on $E$ such that its domain of uniform convergency is exactly equal to $\stackrel{\circ}{G}(E)$.

(iv) Let $D$ be a bounded circular domain starlike with respect to 0 . Let $E$ denote the boundary of $D$. Then the envelope of holomorphy of $D$ is equal to $\stackrel{\circ}{G}(E)$.

\section{Applications of $\Phi(z, E, b)$.}

(a) Application to the theory of interpolation and approximation of holomorphic functions of several variables by polynomials. In the case of one variable, the function $\log \Phi(z, E, 0)$ is a generalized Green's function for the unbounded component of $O E$ with pole at $\infty$ [3]. It is well known that Green's function plays the primary role in the theory of interpolation and approximation of holomorphic functions of one variable by polynomials (see [18]). It turns out that the function $\Phi(z, E, 0), z \epsilon C^{n}$, also plays quite a similar role in the theory of functions of $n$ complex variables. For instance, one obtains the Bernstein-Walsh inequality

$$
\left|P_{v}(z)\right| \leqslant\left(\max _{z \in E}\left|P_{v}(z)\right|\right) \cdot \Phi^{\nu}(z, E, 0), \quad z \epsilon C^{n},
$$

which is used in the proof of the following theorems [16]:

(i) If $\Phi(z, E, 0)$ is continuous in $C^{n}$ and $E_{R}$ is given by

$$
E_{R}=(z: \Phi(z, E, 0)<R), \quad R>1,
$$

then the necessary and sufficient condition that $f(z)$ be holomorphic in $\boldsymbol{E}_{R}$ and not continuable to holomorphic function in any $E_{R^{\prime}}, R^{\prime}>R$, is that

$$
\limsup _{p \rightarrow \infty} \sqrt[p]{\max _{z \in E}\left|f(z)-\pi_{\nu}(z)\right|}=1 / R,
$$

where $\pi_{\nu}(z)$ denotes a polynomial of degree $\nu$ of the Tchebycheff best approsimation to $f(z)$ on $E$.

(ii) $\limsup _{v \rightarrow \infty} \sqrt[v]{\max _{z_{\theta} E}\left|f(z)-L_{v}(z, f)\right|}=\limsup _{v \rightarrow \infty} \sqrt[v]{\max _{z_{\theta} E}\left|f(z)-\pi_{v}(z)\right|} \quad$ where $L_{\nu}(z, f)=\sum_{i=1}^{\substack{\nu \rightarrow \infty \\ \nu}} f\left(q_{i}^{(\nu)}\right) L^{(i)}\left(z, q^{(\nu)}\right), \quad v=1,2, \ldots, \quad$ and $q^{(\nu)}$ is the extremal system (2.2) with respect to $b(z) \equiv \mathbf{0}$.

If $n=1$, these theorems are well known [18].

If $E=E_{1} \times \ldots \times E_{n}$ and $d\left(E_{i}\right)>0(i=1,2, \ldots, n)$, then

$$
\Phi(z, E, 0)=\max \left(\Phi\left(z_{1}, E_{1}, 0\right), \ldots, \Phi\left(z_{n}, E_{n}, 0\right)\right), \quad z \epsilon C^{n} .
$$

This equation implies that the approximation or interpolation of a function $f(z)$ holomorphic in the Cartesian product of plane sets reduces, in principle, to approximation or interpolation in each variable separately.

For instance, if $E=E_{1} \times \ldots \times E_{n}$, then (5.2) is a necessary and sufficient condition that the function $f(z)$ be holomorphic in the Cartesian product $E_{1 R} \times \ldots \times E_{n R}$, where $E_{k R}=\left(z_{k}: \Phi\left(z_{k}, E_{k R}, 0\right)<R\right)$. In the case that $E_{k}, k=1,2, \ldots, n$, is a line segment, this fact has been proved by a different method by Sapogor [13].

(b) Application to the generalized Dirichlet problem for plurisubharmonic functions. The application of $\Phi$ to the generalized Dirichlet problem is based on the following

LEMma. If $\Phi(z, E, 0)$ is finite at any point $z \epsilon O^{n}$, then there exists a finite limit

$$
u(z, E, b)=\lim _{\lambda \downarrow 0} \frac{1}{\lambda} \log [\Phi(z, E, \lambda b) / \Phi(z, E, 0)], \quad z \in C^{n},
$$


and the function

$$
u^{*}(z)=\limsup _{z^{\prime} \rightarrow z} u\left(z^{\prime}, E, b\right)
$$

is plurisubharmonic (for the definition of plurisubharmonic function see [1]) at any interior point of

$$
\mathscr{E}_{1}=(z: \Phi(z, E, 0)=1) .
$$

In the case of one variable the function $u(z, E, b)$ is harmonic in $C E$ and it gives a generalized solution of the Dirichlet problem (in the class of harmonic functions) for $C E$ and boundary values $b(z)$ (see [17] or report [5] by Górski). The connection of $u(z, t, b), z \epsilon C^{n}, n=2$, with Bremermann's generalized solution of the Dirichlet problem is given by the following theorem [16]:

Suppose that for the domain $D$ there exists a sequence of domains of holomorphy $D_{v}$ such that

$$
D_{v} \supset D_{v+1} \supset \bar{D}, \quad v=1,2, \ldots,
$$

and for any $\varepsilon>0$ there is $\nu_{0}$ such that

$$
D_{v} \subset D_{\varepsilon}=\left(z: \min _{\tau_{\epsilon} D}\|z-\zeta\|<\varepsilon\right), \quad v \geqslant v_{0} .
$$

Suppose every function $f(z)$ holomorphic in $\bar{D}$ can be uniformly approximated in $\bar{D}$ by polynomials. If $E$ denotes the silov boundary of $D$ with respect to the holomorphic functions in $\bar{D}$, then the function $u^{*}(z)$ is equal to the upper envelope of all the functions $V(z)$ that are plurisubharmonic in $D$, continuous in $D+E$ and less than or equal to $b(z)$ on $E$, i. e. $u^{*}(z)$ is the Bremermann generalized solution of the Dirichlet problem for $D$ with boundary values $b(z)$.

The proof of the theorem is based on some lemmas on the uniform convergence of Hartogs series whose terms are uniformly bounded on $D$. Those lemmas correspond to the theorems (i)-(iii) of section 4.

To end this report let us add that the extremal points (2.2) with respect to $b(z) \equiv 0$ have been applied in [14] to a generalization of a result of Pólya on analytic continuation of functions of one variable to the case of many variables.

\section{REFERENOES}

[1] H. J. Bremermann, On a generalized Dirichlet problem for plurisubharmonio functions and pseudoconvex domains, Transactions of the American Mathematical Society 91 (1959), p. $246-276$.

[2] M. Fekete, Über die Verteilung der Wurzeln bei gewissen algebraischen Gleichungen mit ganzzahligen Koeffizienten, Mathematische Zeitschrift 17 (1923),
[3] J. Gorski, Sur l'équivalence de deux constructions de la fonction de Green généralisée, Annales de la Société Polonaise de Mathématique 21 (1949), p. 70-73.

[4] - The method of extremal points and Dirichlet's problem in the space of two complex variables, Archive for Rational Mechanics and Analysis 4 (1960), p. $412-427$.

[5] - Some applications of the method of extremal points in the theory of analytic functions of one complex variable, Colloquium Mathematicum 11 (1964)

[6] F. Leja, Sur les séries des polynômes homogènes, Rendiconti del Circolo Matematico di Palermo 56 (1932), p. 412-445.

[7] - Sur les suites de polynômes, les ensembles fermés et la fonction de Green, Annales de la Société Polonaise de Mathématique 12 (1934), p. 57-71.

[8] - Sur une famille des fonctions harmoniques dans le plan liées $\grave{a}$ une fonction donnée sur la frontière d'un domaine, Bulletin International de l'Academie Polonaise des Sciences et des Lettres, Série A, 1936, p. 79-92.

[9] - Sur une olasse de fonctions harmoniques et les séries de Taylor de fonctions de deux variables, Annales de la Société Polonaise de Mathématique 22 (1949), p. $245-268$.

[10] - Teoria funkcji analitycznych, Warszawa 1957 (in Polish).

[11] - Problèmes a résoudre posés à la Oonférence, Colloquium Mathematicum 7 (1959), p. 153.

[12] G. Pólya, Über gewisse notwendige Determinantenkriterien für die Fort. setzbarkeit einer Potenzreihe, Mathematische Annalen 99 (1928), p. 687-706.

[13] Н. А. Сапогов, O наилучием приближсении аналитических функиий нескольких переменных и о рлдах многочленов, Математический Сборник 38 (1956),

[14] M. Schiffer and J. Siciak, Transfinite diameter and analytic continuation of functions of two complex variables, Technical Report, Stanford 1961.

[15] J. Siciak, On an extremal function and domains of convergence of series of homogeneous polynomials, Annales Polonici Mathematici 10 (1961), p. 297-307.

[16] - On some extremal functions and their applications in the theory of analytic functions of several complex variables, Technical Report, Stanford 1961 (also Transaction of the American Mathematical Society 105 (1962), p. 322-357).

[17] - Some applications of the method of extremal points, Colloquium Mathematicum 11(1964), p. 209-250

[18] J. L. Walsh, Interpolation and approximation, New York 1935. p. $228-249$. 